Academic Platform Journal of Engineering and Science

\title{
An Expert System to Predict Eye Disorder Using Deep Convolutional Neural Network
}

\author{
*1,2 Moahmmed Rashid Ahmed, ${ }^{3}$ Adil Deniz Duru, ${ }^{4}$ Osman Nuri Uçan, ${ }^{5}$ Oğuz Bayat \\ *11 Information Technology, Altinbas University, Knt.2093@yahoo.com, (iD \\ ${ }^{2}$ Computer Science, Tikrit University, saadaljanabi95@gmail.com , \\ ${ }^{3}$ Physical Education and Sports Teaching, Marmara University, deniz.duru@marmara.edu.tr, \\ ${ }^{4}$ Electrical and Electronics Engineering, Altinbas University, osman.ucan@altinbas.edu.tr , \\ ${ }^{5}$ Electrical and Electronics Engineering, Altinbas University, oguz.bayat@altinbas.edu.tr, \\ Research Paper \\ Arrival Date: 21.05 .2020 \\ Accepted Date: 17.12.2020
}

\begin{abstract}
Glaucoma according to the W.H.O is one of the major causes of blindness worldwide. Due to its complexity and silent nature early detection of this disease makes it hard to detect. There have been several techniques over the years for classification which have shown significant improvement over the past decade or two. Some of the many classification models are SVM (support vector machine), KNN (K- Nearest Neighbors), Decision tree, Logistic Regression and ANN (Artificial Neural Network) back propagation. For this paper we would consider different procedure and method of early detection of the glaucoma disease using the MATLAB Deep Convolutional Neural Network (DCNN). The DCNN based expert system basically works like the human brain with input, neurons, hidden layers and output. For this project Fundus image of both healthy image and glaucoma image are collected with good lighting condition so that all hidden features can be identify. The Fundus image are then passed through different image processing method such as Grayscale, B\&W, Complement, Robert, Resize and power Transform. The fundus is then passed through a texture feature extraction algorithm know as Deep Convolutional Neural Network (DCNN). The features gotten are Contrast, Correlation, energy, Homogeneity, Entropy, Mean, Standard deviation, Variance, skewness and Kurtosis. After the feature extraction the data are arrangement on a spreadsheet which serves as a means of record. Lastly, a deep convolutional neural network is written with one hidden layer, 16 input neuron and 2 output either healthy or not. The data are split into train and test dataset with $70 \%$ for training $15 \%$ validation and $15 \%$ for testing. Accuracy of detection was $92.78 \%$ with the execution time of 5.33 s only depending on the number of iteration or epochs.
\end{abstract}

Keywords: Expert system, DCNN, glaucoma, deep learning, eye disease, fundus images.

\section{INTRUDUCTION}

Statistic according to the World Health Organization shows that over 286 million people are affected with one form of eyes disease or the other. While 39 million are totally blind and 246 partially. Glaucoma being a major cause of blindness in the world today damages the optic nerves causing partial or total blindness depending on its stage. The eye disease is caused by increasing intraocular pressure (IOP) as explained in [1]. Also, other causes can be traced to heredity, ethnic background and having high myopia. It is also caused by poor blood flow which is meant to regulate the optic nerve. This disease is usually detected late due to the fact that it's usually painless until it gets to critical state as said in [2]. So, the ever-growing need for quick and automated detection is needed in most societies today.

In this paper we are going to introduce a system with a GUI that takes in images. Extracts features of the image passed into it and perform different image processing techniques. This project would certainly be helpful in early Glaucoma detection and would cost less for such test to be perform on patients of all kinds. With this paper health care inflation would slow down, elimination of waste and increase in efficiency thereby reducing production cost would be experience in most hospitals.

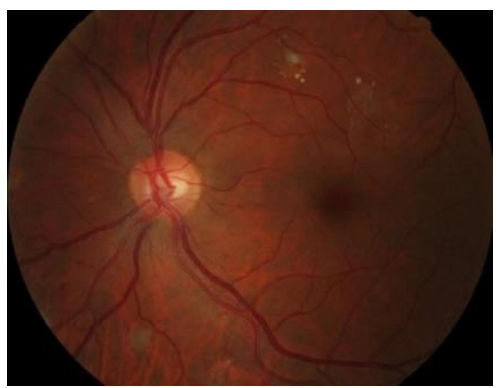

Figure 1. Glaucoma Fungus image 


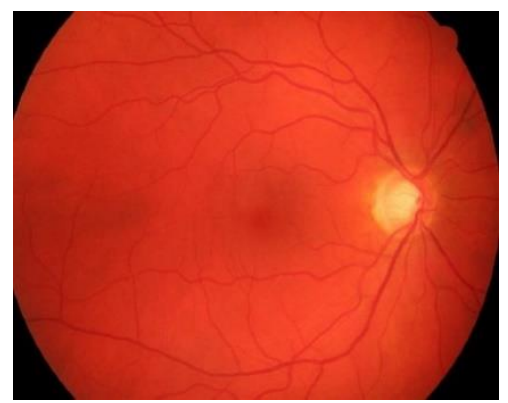

Figure 2. Healthy Fungus Image

The figure 1 and 2 are the fundus image of both and healthy and glaucoma image which would be later passed through the DCNN algorithm to get necessary features. The goal of the project is to help design a system that is much faster and efficient in making accurate prediction. NOTE: this expert system is not aimed at replacing scientists in this filed.

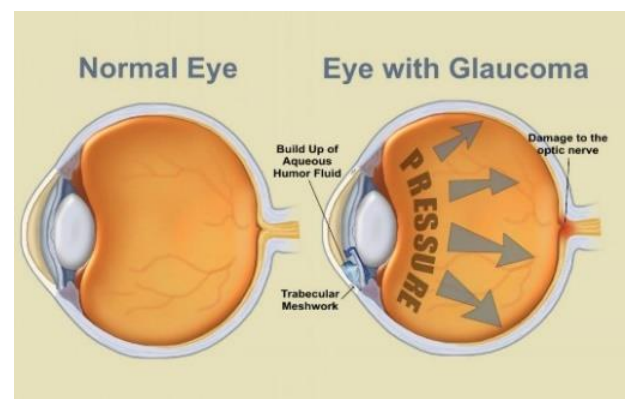

Figure 3. Pressure in Non-glaucoma and Glaucoma eyeball [3].

\subsection{Problem Statement}

The challenge inherent in biomedical information systems and eye diseases detection from detecting very reversed disease Glaucoma. Due to its growing complexity, this disease often causes serious damage to optic nerves that can be found and detected. However, there is no such automated expert system available for detecting glaucoma using deep learning techniques. Commonly referred to as a worst eye disease, these types of diseases can be very damaging and frustrating to humans. Therefore, as mentioned previously, the best strategy is to implement an expert system that could expect the inevitable result that with enough time and resources, an eye will inevitably gain access to the detection of glaucoma. It is paramount that when this occurs, the detection is discovered promptly and quarantined or eliminated before any material harm is done.

\subsection{Aim of Study}

- This research thesis approaches the challenge of detecting glaucoma eye disease using deep convolutional neural network in a two-fold manner.

- First, a fully connected Deep convolutional neural network (DCNN) is used to train on Fundus Images with supervised learning using labeled healthy and glaucoma disease.
- Kaggle dataset will be used which is more representative of modern-day eye disease for an expert system and do not have drawbacks of previous datasets commonly used in the field.

- We want to achieve a learning patterns of detecting eye disease by training a fully connected deep convolutional neural network for expert system

- The expert system can reliably and effectively detect glaucoma eye disease with a high degree of accuracy, high rate of recall, and a low rate of false positive rate.

- The expert system is considered to be a form of patternbased detection because the system is trained on known well and known bad patterns and taught to detect these diseases in future, unseen flows using deep learning.İçerik 2 sütun halinde Times new roman olarak 10 punto halinde yazılmalıdır.

\section{BACKGROUND}

Great effort has been made by researchers all over the world and numerous studies have been presented over a period of years [4]. The fundus image is usually presented in RGB form, which are then converted to grayscale to make easy processing of the image. This is done become RGB are usually $3 x$ the size of Grayscale image as described in [5]. Then different image processing technique are performed on the image to help understand the image better as differentiated in [6]. The GLCM feature extraction technique is then performed to get some feature after that passed to a neural network back propagation as given in [7].

Optic disk is very important in RGB of fundus image. As the optic disk extract features from ROI (region of interest). Also, Glaucoma is detected by the appearance of hemorrhages on optic disk.

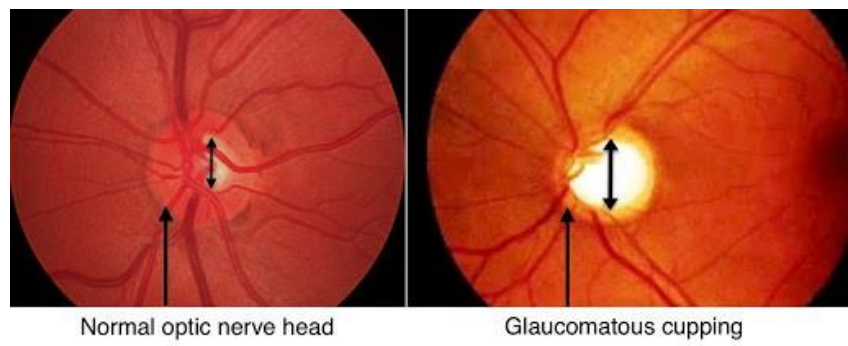

Figure 4. Optic nerve head of non-glaucoma and glaucoma eye [7].

Other faster ways of detection are applying convolutional neural network technique. This method works the same way the optical human eyes function. Image is first converted into pixels, this process is called convolution, after that it is passed to the hidden layer where max-pooling and fullyconnected layer is form as described in [8]. The number of hidden layers depend on the data and how strong your computing power can process as much data as possible[9]. It is said to get a higher accuracy the number of hidden layers and neurons have to be increased. Dropout are usually added to prevent over-fitting of the model as updated by 10]. The DCNN network would be shown in further updates. 
Researcher in [11] had a breakthrough a couple of years ago with the Concept of Transfer learning which otherwise known as pre-trained model with optimization. In this type of architecture neural network does not need to be built from stretch but rather an already built network is used with only the output removed. This technique was achieved during a competition organized yearly by ImageNet, with each competitor coming up with different models and architecture to see who have the best model with close to 100's of hidden layers as described by author in [12].

It should be noted that the transfer learning technique does not require much data as the pre-trained data contains millions of data with thousands of categories. Only the output sigmoid layer is removed and changed to your preferred output.

\section{METHODOLOGY}

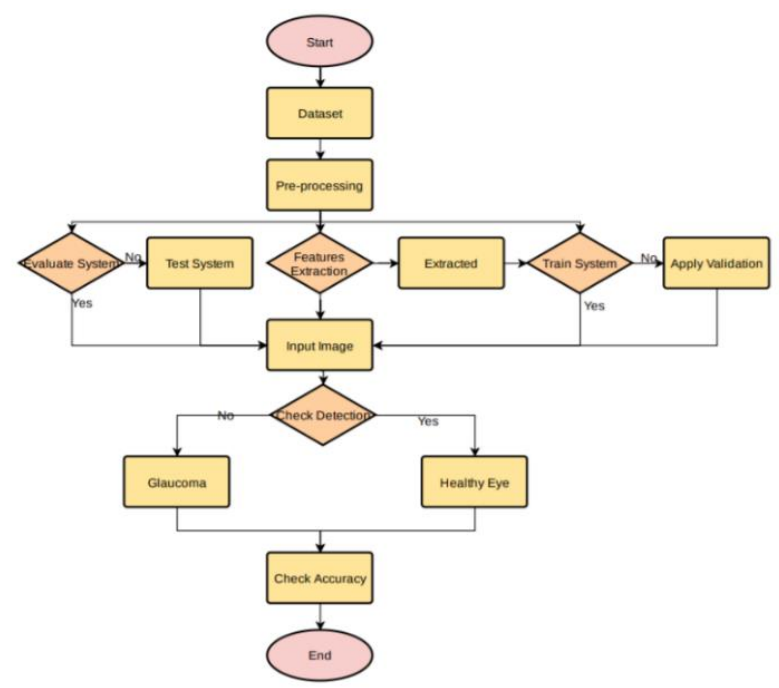

Figure 5. Flowchart shows an overall approach being followed.

The glaucoma eye disease detection is based on extracting features using the from fundus images. The different steps carried out would be shown on a flowchart, which gives a better understanding on steps carried out. This work will fill the gap of research where a separate model for each fundus image for eye disease was been created. The motivation is that training, deploying and maintaining an expert system for glaucoma eye disease could quickly become burdensome in big networks especially for deep learning systems. This work focuses on using newer benchmark dataset that have recently become available to the research community, the Kaggle Dataset [12] will be used using deep convolutional neural network on MATLAB R2018b. This deep convolutional neural network will have similar size and number of layers and their formula for the threshold will be used, albeit it will be modified - a parameter will be added to it, and an optimization is made for this parameter. Some details, such as activation function choice and optimization algorithm choice are this work's original contributions. Additionally, this work will employ a feature selection mechanism and will predict the accuracy of feature selection parameters efficiently using deep learning. This work will allow for assessment of deep convolutional neural network method accuracy on smaller feature subsets and for making a fairer comparison on eye diseases detection and classification for glaucoma with more advanced deep learning method.

\subsection{Dataset Details}

Kaggle Dataset deals with the process and form at which data are usually collected from a repository known as Kaggle. Data can either be in form of .JPEG or MPEG. But for this research the fungus images are in .JPEG form and are two folders both for the healthy and Glaucoma image. The most useful datasets for Glaucoma fundus images are those containing captures of real network environments. This dataset is easily shared with the public, as they contain details of Glaucoma and Healthy eyes, and more importantly sensitive information about the fundus images for expert system on the respective network. Furthermore, the effort required to create a labeled dataset from the raw network traces is an immense undertaking. As a consequence, researchers often resort to optimal dataset that can be shared amongst the research community on Kaggle.

\subsection{Deep Convolutional Neural Network}

Deep Convolutional Neural Network (DCNN) architecture and hyperparameters refer to the parameters that are set before the learning process begins. These DCNN hyperparameters are tuned based on a feedback loop of the model's performance on the validation set.

Throughout the training and validation cycle, these hyperparameters are tuned in order to achieve highest and best performance for the deep learning model. The most effective neural network architecture and hyperparameters used in this work.The configuration for the deep convolutional neural network gave best results in the experiments. The first hidden layer for the continuous input features is much larger in this case at 128 neurons, than the number of inputs at 7 . Smaller numbers of neurons were also tested, and found to achieve comparable results. Therefore, to keep architectures consistent, this same general configuration is also used in the study with Kaggle Dataset, which has many more continuous input features.

Hyperparameters fall into the following main categories:

- Layer size, number of units per layer

- Magnitude (momentum, learning rate)

- Regularization (dropout, L1, L2)

- Activation functions (sigmoid, relu, tanh, etc.)

- Weight initialization

- Loss functions

- Number of epochs to train, and batch size per epoch

- Data normalization scheme 


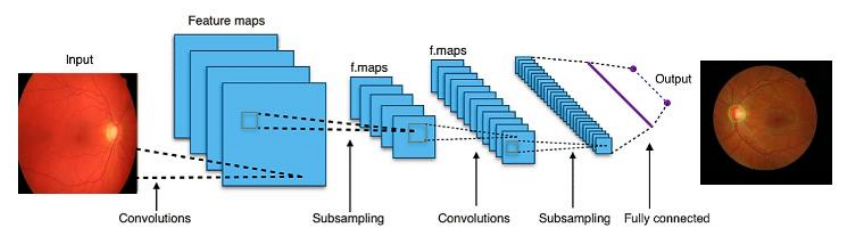

Figure 6. An architectural diagram of deep convolutional neural network from input leading to output different fullyconnected layers for processing glaucoma.

\subsection{Image Pre-Processing}

Image processing basically deals with pixels adjustment. Adjusting the pixels of an image to a desired form. Data preprocessing are usually done to reduce contrast, unwanted noise of image, luminous of the image. For the preprocessing aspect the image is converted to grayscale, Black and White, Histogram Equalization and Thresholding.

More image pre-processing technique were carried out on the image using a Developed GUI on the MATLAB software.
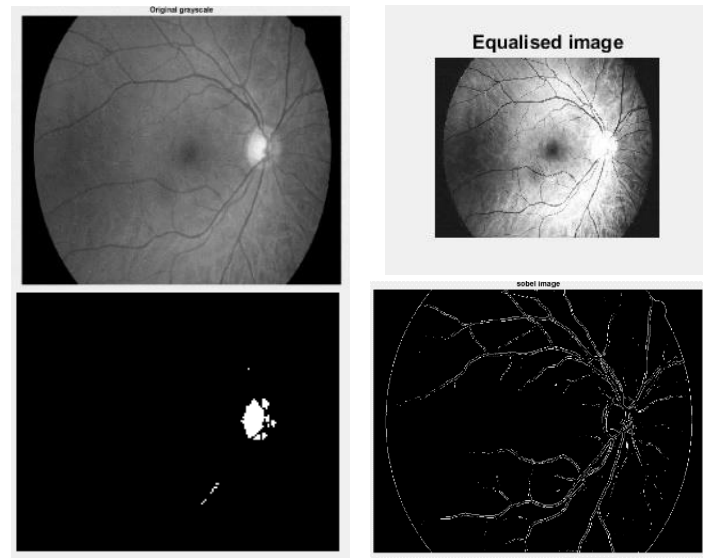

Figure 7. Thresholding applied on images.

\subsection{Feature Extraction}

Feature extraction is an important process before training and testing phase. This process involves extracting important features from the image dataset. This feature includes: Contrast, Correlation, Energy, Homogeneity and Entropy. The GLCM is a technique that evaluates texture of image by taking into attention the spatial relationship of pixels.

In this research feature extraction is important to easy identify between the Glaucoma and healthy eyes. The matrices distance is usually in degree of various form which are starting from $0,45,90$, and 135 . To get a better accuracy we can increased the number of features gotten to be more.

\subsection{Training and Testing}

The dataset gotten was split into three parts $70 \%$ training, $15 \%$ testing and $15 \%$ Validation. A deep convolutional neural network back propagation was created using the MATLAB nntool. With a total of 60 epochs each was set.
The number of accuracies can also be increased if we retrain the neural network repeatedly.

The input data are set at 5 different input. With a single hidden layer, a total of 16 neuron are set in the hidden layer with 2 outputs. After the neural network have been created, the gotten feature that are extracted from the image are then passed into the network for easy classification between healthy or glaucoma. Note that we had to represent the string data in numerical form.

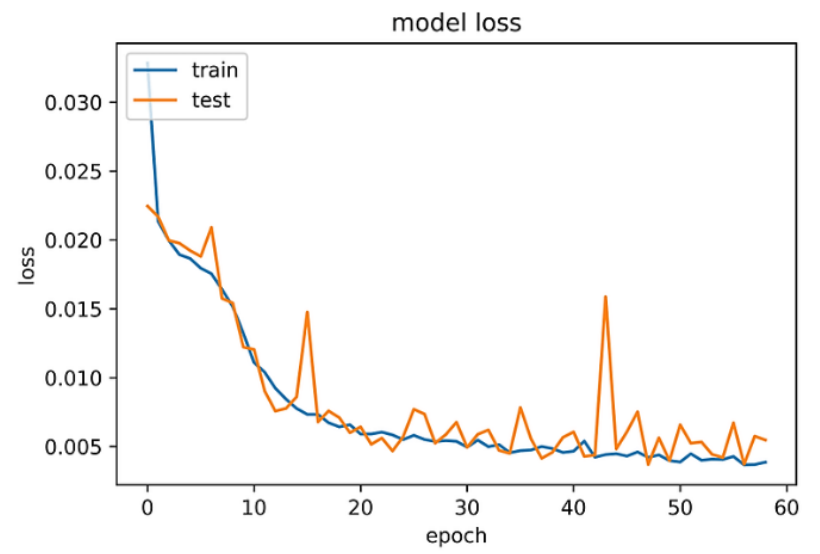

Figure 8. The training and testing parameters for deep convolutional neural network with respect to model loss and 60 -epochs training.

\section{RESULTS}

In this section, we have considered two parameters while analyzing the deep learning algorithm (DCNN) on glaucoma samples for an accuracy in terms of percentage and time in terms of seconds. The experiment is carried using MATLAB R2018b Windows environment on 12 GB RAM and $3.2 \mathrm{GHz}$ Intel Core i7 with 4 GB Nvidia GPU. After carrying out several experiments using the deep convolutional neural network, we have come to present all our findings here. We carried out deep convolutional neural network algorithms on our dataset. The results are shown as below. We have done the test procedure with $15 \%$ data which is equal to 13583 data. The deep convolutional neural network was created and trained on all features. Training was limited to 60 epochs with additional condition of early stopping, using functionality provided by deep learning toolbox. This ensures that the model is trained only until the score on validation set is not getting worse - this in turn helps to avoid overfitting the training set.

The deep convolutional neural network is trained to reconstruct inputs from a latent space representation based on only glaucoma and healthy eyes. Therefore, when it receives healthy eye sample as input, it is easier for the deep convolutional neural network to detect the eye, thus resulting in a higher accuracy. When there is sufficient amount of glaucoma in an eye sample image, a deep convolutional neural network take some more time to process and can be used to detect anomalous region by passing through whole pipeline of processing. 

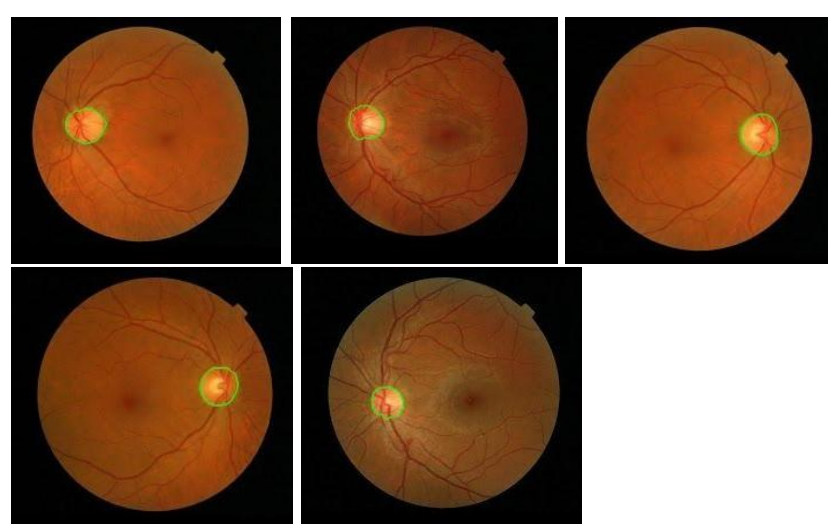

Figure 9. Final Output from the DCNN model detecting Glaucoma Disease Optic Centre of the fundus image

Table 1. The above table contains the sample image and the risk of the patient to confer to Glaucoma.

\begin{tabular}{|c|c|}
\hline Sample Image & Glaucoma Risk \\
\hline V0001.jpg & 0.28218 \\
\hline V0002.jpg & 0.34816 \\
\hline V0003.jpg & 0.027847 \\
\hline V0004.jpg & 0.28081 \\
\hline V0005.jpg & 0.28086 \\
\hline V0006.jpg & 0.77814 \\
\hline V0007.jpg & 0.23869 \\
\hline V0008.jpg & 0.35365 \\
\hline V0009.jpg & 0.28825 \\
\hline V0010.jpg & 0.24779 \\
\hline V0011.jpg & 0.018874 \\
\hline V0012.jpg & 0.22376 \\
\hline V0013.jpg & 0.23201 \\
\hline V0014.jpg & 0.3038 \\
\hline V0015.jpg & 0.24816 \\
\hline V0016.jpg & 0.24682 \\
\hline V0017.jpg & 0.37032 \\
\hline V0018.jpg & 0.39568 \\
\hline V0019.jpg & 0.24506 \\
\hline V0020.jpg & 0.6983 \\
\hline
\end{tabular}

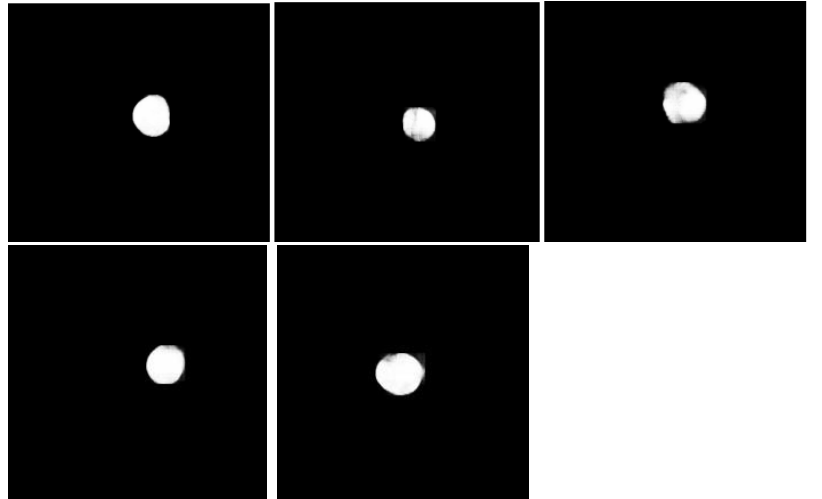

Figure 10. Masked Layers from DCNN Model for the detected Optic Centre of glaucoma disease.

Figure 11. The segmentation done by the model.

\section{DISCUSSION}

Convolutional Neural Network structures have been widely well studied in the discrimination of healty subjects from controls or identfying different mental states of the subjects based on medical images in the literature $[15,16]$.

Deep Convolutional Neural Networks (DCNN) are effective when working with imagery data that consists of lots of examples, and with categorical variables of high cardinality, which are present in the domain of biomedical data.

Detecting glaucoma for the leverages and power of deep convolutional neural networks to achieve better results than other leading techniques without needing to perform much in the way of hand-engineered features, especially when the amount of training data is larger. Furthermore, it was found that using the glaucoma features as an embedded categorical feature enabled the best performance for the deep convolutional neural network. It is theorized that by using the DCNN technique and updating the weights of the glaucoma representation at each epoch, the DCNN forms a type of inherent memory about the disease in relation to the other features and the given label. Additionally, high performance was achieved when using the devised DCNN architecture for detection.

\begin{tabular}{|c|c|c|}
\hline ARTICLE & TECHNIQUE & ACCURACY \\
\hline$[14]$ & Decision Tree & $85.81 \%$ \\
\hline$[14]$ & Naive Baise & $81.53 \%$ \\
\hline$[14]$ & Random Forest & $86.63 \%$ \\
\hline$[14]$ & Neural Network & 85.98 \\
\hline Proposed & $\begin{array}{c}\text { Deep Convolutional } \\
\text { Neural Network }\end{array}$ & $95.77 \%$ \\
& \multicolumn{2}{|}{} \\
\hline
\end{tabular}




\section{CONCLUSION}

In this novel research work, we have developed an intelligence expert system for the purpose of detection of most dangerous eye disease using deep convolutional neural network. Deep learning methods demonstrated good detection and accuracy. For the training, testing and validation of Kaggle based dataset for eye diseases, a wellknown MATLAB R2018b software was used for this purpose. A deep convolutional neural network is written with one hidden layer, 16 input neuron and 2 output either healthy or not. The data are split into train and test dataset with $70 \%$ for training $15 \%$ validation and $15 \%$ for testing. Accuracy is found to vary between $91-92 \%$ depending on the number of iteration or epochs. Moreover, DCNN method can work well with varying number of features and generally its performance for detection purpose does not suffer from extra features, meaning that in real world environment they offer the possibility of using all existing data features. It was also shown that there may not be a need to create different models for eye disease detection, and that one model trained on data can detect glaucoma disease efficiently. This works shows that the overall accuracy of deep learning models can mitigated to some degree with deep convolutional neural network technique and achieving the accuracy of up to $92.78 \%$ with the execution time of 5.33 s only.

\section{REFERENCES}

[1].Xu, Y., Lin, S., Wong, T.Y., Liu, J., Xu, D.: Efficient ReconstructionBased Optic Cup Localization for Glaucoma Screening. In: MICCAI 2013

[2]. C. Bowd, R. N. Weinreb, J. M. Williams, and L. M. Zangwill, "The retinal nerve fiber layer thickness in ocular hypertensive, normal, and glaucomatous eyes with optical coherence tomography," Archives of ophthalmology, vol. 118, no. 1, pp. 22-26, 2000.

[3]. Space healthcare. Understanding glaucoma. http://space-healthcare.com/wpcontent/uploads/2015/02/glaucoma-300x197.jpg.

[4]. Hinton, G.E., Srivastava, N., Krizhevsky, A., Sutskever, I., Salakhutdinov, R.R.: Improving neural networks by preventing co-adaptation of feature detectors. In: Arxiv 2012 [5]. Ciresan, D.C., Meier, U., Masci, J., Gambardella, L.M., Schmidhuber, J.: High-performance neural networks for visual object classification. In: Arxiv 2011

[6]. Xu, Y., Xu, D., Lin, S., Liu, J., Cheng, J., Cheung, C.Y.,
Aung, T., Wong, T.Y.: Sliding Window and Regression based Cup Detection in Digital Fundus Images for Glaucoma Diagnosis. In: MICCAI 2011

[7]. R. R. Selvaraju, M. Cogswell, A. Das, R. Vedantam, D. Parikh, and D. Batra, "Optic nerve head of non-glaucoma and glaucoma eye via gradient-based localization," in Proceedings of the IEEE International Conference on Computer Vision, pp. 618-626, 2017.

[8]. P. J. Foster, R. Buhrmann, H. A. Quigley, and G. J. Johnson, "The definition and classification of glaucoma in prevalence surveys," British journal of ophthalmology, vol. 86, no. 2, pp. 238-242, 2015.

[9]. S. Ahmed, O. Uçan, Adil Deniz Duru, Oğuz Bayat, "Breast Cancer Detection And Image Evaluation Using Augmented Deep Convolutional Neural Networks", Aurum Mühendislik Sistemleri ve Mimarlık Dergisi, Vol. 2, No. 2, Pp. 121-129, 2018.

[10]. J. De Fauw, J. R. Ledsam, B. Romera-Paredes, S. Nikolov, N. Tomasev, S. Blackwell, H. Askham, X. Glorot, B. ODonoghue, D. Visentin, et al., "Clinically applicable deep learning for diagnosis and referral in retinal disease," Nature medicine, vol. 24, no. 9, p. 1342, 2018.

[11]. F. Iandola, M. Moskewicz, S. Karayev, R. Girshick, T. Darrell, and K. Keutzer, "Densenet: Implementing efficient convnet descriptor pyramids," arXiv preprint arXiv:1404.1869, 2014.

[12]. Y. Wu and K. He, "Group normalization," in Proceedings of the European Conference on Computer Vision (ECCV), pp. 3-19, 2018.

[13]. Available Online: https://www.kaggle.com/kondwani/eye-disease-dataset.

[14]. Malik, S.; Kanwal, N.; Asghar, M.N.; Sadiq, M.A.A.; Karamat, I.; Fleury, M. Data Driven Approach for Eye Disease Classification with Machine Learning. Appl. Sci. 2019, 9, 2789.

[15]. D. G. Duru and A. D. Duru, "MR-MS Image Classification based on Convolutional Neural Networks," 2019 Scientific Meeting on Electrical-Electronics \& Biomedical Engineering and Computer Science (EBBT), Istanbul, Turkey, 2019, pp. 1-4, doi: 10.1109/EBBT.2019.8741752.

[16]. D. G. Duru and A. D. Duru, "Classification of Event Related Potential Patterns using Deep Learning," 2018 Medical Technologies National Congress (TIPTEKNO), Magusa, 2018, pp. 1-4, doi: 10.1109/TIPTEKNO.2018.8597016. 